\title{
Biomechanical comparison between single- bundle and double-bundle anterior cruciate ligament reconstruction with hamstring tendon under cyclic loading condition
}

\author{
Shuya Nohmi, Yasuyuki Ishibashi", Eiichi Tsuda, Yuji Yamamoto, Harehiko Tsukada and Satoshi Toh
}

\begin{abstract}
Purpose: The purpose of this study was to compare the anterior tibial translation (ATT) of the anterior cruciate ligament (ACL) reconstructed-knee between single-bundle and double-bundle $A C L$ reconstruction under cyclic loading.

Methods: Single-bundle and double-bundle reconstructions of the knee were performed sequentially in randomized order on the same side using eight human amputated knees. After each reconstruction, the reconstructed-knee was subjected to 500-cycles of 0 to 100-N anterior tibial loads using a material testing machine. The ATT before and after cyclic loading and "laxity increase", which indicated a permanent elongation of the graft construct, was also determined.

Results: The ATT after cyclic loading increased in both single-bundle and double-bundle reconstruction techniques compared to that without cyclic loading. Changes in ATT before and after cyclic loading were $3.9 \pm$ $0.9 \mathrm{~mm}$ and $2.9 \pm 0.6 \mathrm{~mm}$ respectively, and were significantly different. Laxity increase was also significantly different $(4.3 \pm 0.9 \mathrm{~mm}$ and $3.2 \pm 0.8 \mathrm{~mm}$ respectively). Although no graft rupture or graft fixation failure was found during cyclic loading, the graft deviated into an eccentric position within the tunnel.

Conclusions: Although ATT was significantly increased in both single-bundle and double-bundle reconstruction with hamstring tendon after cyclic loading test, there was significant difference. Double-bundle reconstruction might be superior to prevent increasing ATT under cyclic loading. Deformation of hamstring tendon after cyclic loading might result in deterioration of knee stability after ACL reconstruction, and is one of disadvantages of soft tissue graft.
\end{abstract}

\section{Introduction}

The anterior cruciate ligament (ACL) is a commonly injured ligament within the knee joint, and arthroscopic single-bundle (SB) ACL reconstructions have been performed worldwide using biological substitutes, such as bone-patella tendon- bone autograft, hamstring tendon autograft, and allograft. Although most patients have their normal knee function restored and can return to their pre-injury activity level after SB reconstruction, some patients still feel instability even though their reconstructed knee is stable as measured by Lachman

\footnotetext{
* Correspondence: yasuyuki@cc.hirosaki-u.ac.jp

Department of Orthopaedic Surgery, Hirosaki University Graduate School of Medicine, 5 Zaifu-cho, Hirosaki, Aomori 036-8562, Japan
}

test and an instrumented knee laxity device such as the KT-1000 arthrometer [1]. This means that traditional SB reconstruction techniques do not completely reproduce the original ACL [2-4], which anatomically and functionally consists of two bundles: the anteromedial (AM) bundle and the posterolateral (PL) bundle [5]. Therefore, double-bundle (DB) ACL reconstructions, which reproduce both the AM and PL bundles, have been developed to improve clinical outcomes. Although several recent Level I and II studies have shown that DB reconstruction had superior short-term results than SB reconstruction [6-10], some studies showed no advantage for DB reconstruction [11], so it is still controversial.

Biomechanical advantages of DB reconstruction were first demonstrated by Radford and Amis [12] using a 
materials testing machine (Instron 1122), which could only apply an anterior-posterior (AP) load. Recently, new robotic technologies have been introduced into the knee biomechanical studies [13]. This technology can apply more complex loads, such as varus/valgus and rotational loads, and has demonstrated the functional role of the AM and PL bundle as well as biomechanical advantages of anatomical DB reconstruction, especially to manipulate a combined rotatory load that mimics the pivot shift test [4,14-16]. Although these robotic studies demonstrate the complex function of the ACL, only a single testing condition or a small number of loads were used. In actuality, implanted graft materials within the knee joint must bear cyclic loading during daily activities and also in postoperative rehabilitation [17-21]. There have been few studies which compare SB and anatomical DB ACL reconstruction under cyclic loading conditions. Therefore, the purpose of this study was to compare the anterior tibial translation (ATT) of the ACL reconstructed-knee under cyclic loading between $\mathrm{SB}$ and $\mathrm{DB}$ ACL reconstruction. It was hypothesized that the DB reconstruction would decrease ATT compared to the SB technique, and would subsequently reduce ATT increase during cyclic loading.

\section{Materials and methods}

Eight fresh-frozen amputated human knees with an average age of 70.6 (18-93) years were used in this study. Specimens had no prior surgery or evidence of abnormal laxity, and obvious degenerative knees were excluded. Informed consent was obtained from the patients and ethical approval of this study was obtained from the Ethics Committee of Hirosaki University School of Medicine. Specimens stored at $-80^{\circ} \mathrm{C}$ were thawed at room temperature 24 hours before testing and kept moist with saline spray during preparations and mechanical testing [22]. Surrounding skin and muscles were removed to expose the bone, leaving the knee ligaments and popliteus muscle and tendon intact. The extensor mechanism was removed because it has no significant effect on anterior-posterior (A-P) drawer motion up to $90^{\circ}$ of flexion [23]. The proximal part of the femur and the distal part of the tibia were placed in custom-made metallic pots of polymethylmethacrylate for gripping to test the fixture's rigidity. The specimens were mounted on a materials testing machine (Instron 4465; Instron Corp, Canton, MA) with custom-made apparatus without restricting five degrees of freedom except for varus-valgus rotation (Figure 1).

\section{Testing protocol}

Before sectioning the ACL, the neutral anterior-posterior (A-P) position of the intact knee at $30^{\circ}$ of knee flexion was determined and used as a reference position (RP)

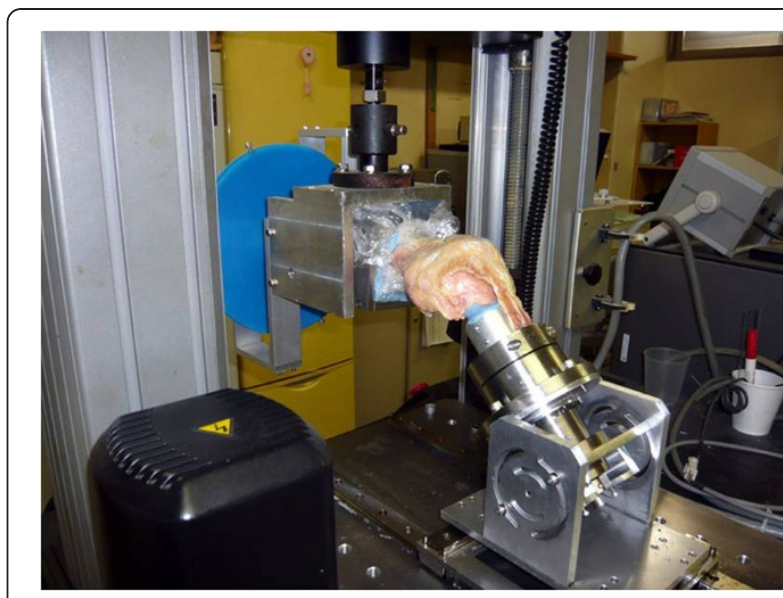

Figure 1 Photograph of the testing system with knee joint at $30^{\circ}$ of flexion.

throughout the testing (Figure 2). It was defined as the position midway between the 2 zero points of the loaddisplacement hysteresis loop by imposing a $\pm 50-\mathrm{N}$ drawer cycle [24] resulting in four degrees of freedom (DOF) knee kinematics (medial-lateral, and proximal-distal translations) (Figure 3). After determining the RP (A-P neutral position), the intact knee was subjected to 30 cycles of 0 to $100-\mathrm{N}$ anterior tibial loads at a crosshead speed of $100 \mathrm{~mm} / \mathrm{min}$ and load-displacement curve was recorded. Displacement of the tibia at $100-\mathrm{N}$ anterior tibial loads from RP was defined as ATT (anterior translation of the tibia), and ATT of the intact knee (after 30 cyclic load) was recorded. Next, the ACL was transected to simulate an isolated ACL tear, and the same cyclic loading conditions were also applied to the ACL-deficient knee and the load-displacement curve was recorded.

$\mathrm{SB}$ and DB-ACL reconstructions were performed sequentially in randomized order in the same knee. Each reconstructed knee was subjected to 500-cycles of 0 to $100-\mathrm{N}$ anterior tibial loads at a crosshead speed of $100 \mathrm{~mm} / \mathrm{min}$ and load-displacement curve was recorded. The ATT in response to an anterior load of $100-\mathrm{N}$ before and after cyclic loading was determined in reference to the neutral A-P position. Additionally, the parameter "laxity increase" introduced by Scheffler et al. [24]. to quantify the loss of graft fixation was calculated as the change in the tibial position at load pickup between the first and last cycle of cyclic loading (Figure 4). The laxity increase is distance between initial tibial position before cyclic loading and the final position after cyclic loading. This causes a permanent elongation of the graft constructs, which consists of the graft slippage from the fixation device and plastic deformation of the linkage materials and knot tightening. Therefore, the ATT consists of the laxity increase and the recoverable elongation of the tendon graft itself. 


\section{Determination of the reference position of the intact knee $\downarrow$ \\ ATT under anterior tibial load of $100-\mathrm{N}$ in the intact knee \\ Cut ACL \\ ATT under anterior tibial load of $100-\mathrm{N}$ in the ACL deficient knee \\ ACL reconstruction \\ ATT under anterior tibial load of $100-\mathrm{N}$ in each reconstructed knee \\ 500 -cycle of 0 to $100-\mathrm{N}$ anterior tibial loads \\ Determine change in ATT and laxity increase}

Figure 2 Testing protocol and data obtained. A-P: Anterior-posterior, AT: Anterior tibial translation from neutral A-P position.

\section{Surgical procedure}

ACL graft materials were semitendinosus and/or gracilis tendons, which were harvested from each knee specimen during preparation and also other amputated human knees, which were used for another study. These tendons were trimmed, and cut in half if the tendon was long enough (over $24 \mathrm{~cm}$ ). Both ends of each graft were connected with No. 2 Ethibond sutures (Ethicon, Inc., Somerville, NJ, US) using baseball glove sutures, and folded once (single loop). To decrease difference between SB- and DB-ACL reconstructions, each procedure used two looped graft (4 strands tendon) totally. However, graft conditions were not the same because grafts were used for

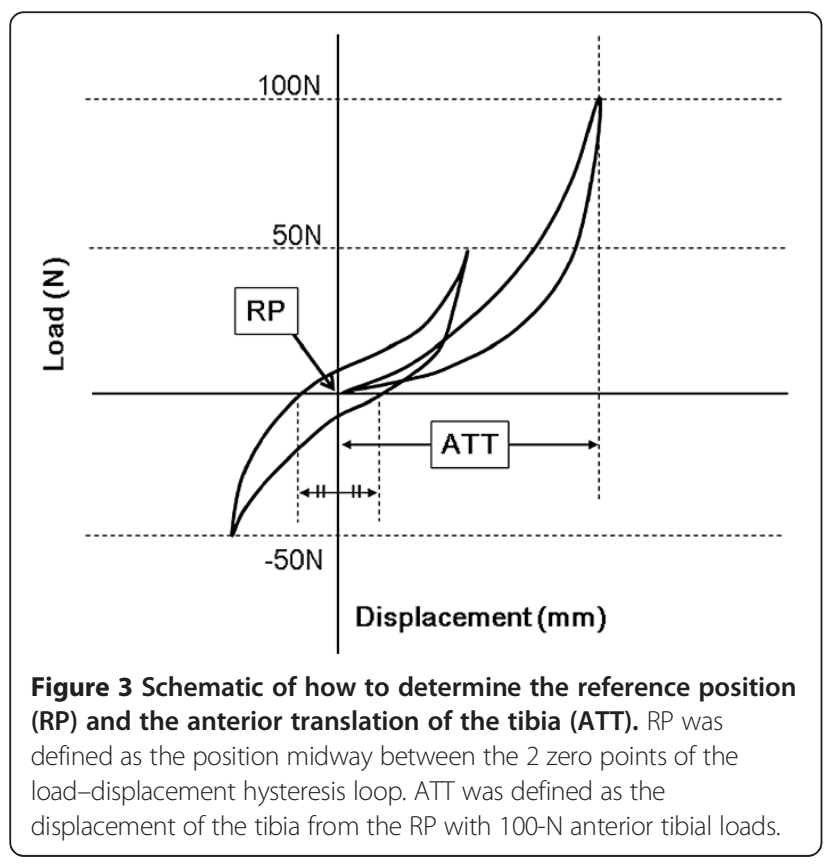

each cyclic loading test. For SB-ACL reconstruction, double looped grafts were combined and used if the diameter was to be more than $6 \mathrm{~mm}(6-8 \mathrm{~mm})$. For DB-ACL reconstruction, single looped grafts were used for the AM bundle and PL bundle graft, and diameters of grafts were usually about $5 \mathrm{~mm}(4.5-6 \mathrm{~mm})$. These grafts were pretensioned with 20-lb during tunnel preparation using the Graft Master II (Smith \& Nephew, Andover, MA, USA). A suture plate (B/Braun AESCULAP, Tuttlingen, Germany) was attached to the proximal end of each graft using No.5 Ethibond, and the length of the suture loop was matched to the femoral tunnel measured during reconstruction.

After cyclic loading test of intact and ACL-deficient knee, each specimen was detached once from the clamp. The ACL remnant was removed and both femoral and tibial footprints of the AM bundle and the PL bundle were carefully identified under direct visualization. Notch plasty was not performed. Then, SB- and DB-ACL reconstructions were performed in randomized order. After the first reconstruction and its testing, the created tunnels were packed by cancellous bone, and then the next reconstruction was performed. These cancellous bones were harvested from another specimen's knee condyle using a coring reamer. Diameters of these coring reamers were $1 \mathrm{~mm}$ larger than the created tunnel. Therefore, the prior tunnel was firmly packed with solid bone to decrease adverse effects on the next reconstruction.

For SB-ACL reconstruction, the femoral and the tibial guide pins were inserted into the center of each footprint (middle point between the AM and PL bundle footprints). These pins were overdrilled outside-in by a cannulated drill matched with graft diameter. For DB$\mathrm{ACL}$ reconstruction, the $\mathrm{AM}$ and $\mathrm{PL}$ guide pins were inserted into the center of the AM and PL bundle footprints with careful attention not to overlap the tunnels. 


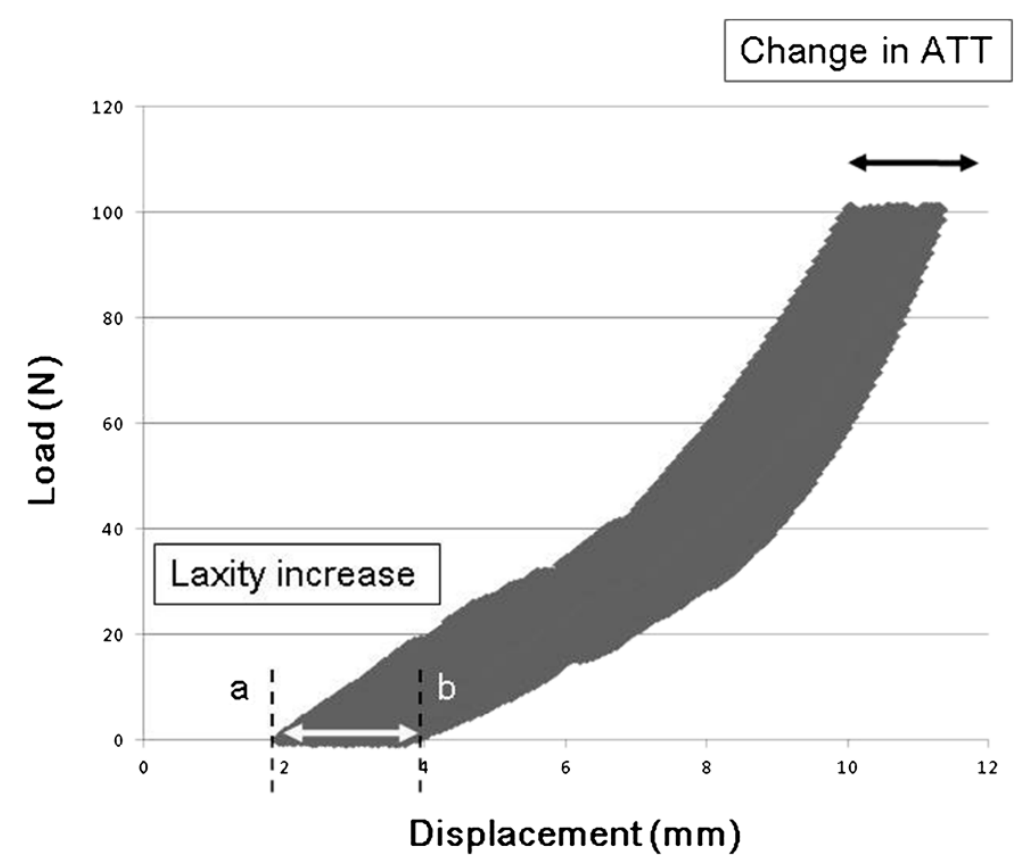

Figure 4 Schematic of change in anterior tibial translation (ATT) and laxity increase in load-displacement curve during cyclic loading. The laxity increase is distance between initial tibial position before cyclic loading and the final position after cyclic loading.

These pins were overdrilled in the same manner as the SB-ACL reconstruction. Grafts for SB- or DB-ACL reconstructions were inserted from proximal (femoral tunnel) to distal (tibial tunnel) antegradely, and then the distal end of each graft was connected with a Double Spiked Plate (DSP; Meira Corp, Nagoya, Aichi, Japan) through No.2 Ethibond [25]. Knees were kept at $30^{\circ}$ of knee flexion on the materials testing machine at the neutral position, and grafts were tensioned using a calibrated spring scale and fixed at 20-lb for SB-ACL reconstruction and 10-lb each (total of 20-lb) in DB-ACL reconstructions through the Double Spiked Plate.

\section{Statistical analysis}

Statistical analyses were performed to compare ATT and laxity increase between SB-ACL reconstructed and DB-ACL reconstructed knees. For these biomechanical data, a paired t test was used. Statistical analyses were also performed to compare the ATT between intact knees, ACL deficient knees, SB-ACL reconstructed and DB-ACL reconstructed knees. For these biomechanical data, statistical evaluation was made using a two-way analysis of variance with the Tukey HSD test for post hoc multiple comparisons. The level of significance was set at $\mathrm{P}<0.05$. SPSS version 12.0 software (SPSS Inc., Chicago, Illinois, USA) was used for statistical analysis in this study.

\section{Results}

ATT in the intact knee $(9.1 \pm 3.9 \mathrm{~mm})$ was significantly increased in the ACL-deficient knee $(16.7 \pm 4.3 \mathrm{~mm})$ $(\mathrm{P}=0.003)$ (Table 1). Before cyclic loading, ATT in the ACL-deficient knee was significantly improved in both SB $(11.8 \pm 3.6 \mathrm{~mm})$ and $\mathrm{DB}$ reconstruction $(10.1 \pm 4.0$ $\mathrm{mm}$ ), and there were no significant differences between the reconstruction techniques $(\mathrm{P}=0.961)$. ATT after cyclic loading increased in both reconstruction techniques compared to that without cyclic loading. ATT after cyclic loading was $15.7 \pm 4.1 \mathrm{~mm}$ in $\mathrm{SB}$ reconstruction $(\mathrm{P}=$ 0.077 , compared with before cyclic loading) and $13.1 \pm$ $3.9 \mathrm{~mm}$ in $\mathrm{DB}$ reconstruction $(\mathrm{P}=0.677)$. There was significant difference in the ATT after cyclic loading between $\mathrm{SB}$ and $\mathrm{DB}$ reconstruction $(\mathrm{P}=0$. 027). Therefore, changes in ATT between before and after cyclic loading were also significantly different $(3.9 \pm 0.9 \mathrm{~mm}$ and $2.9 \pm$ $0.6 \mathrm{~mm}$, respectively) (Figure 5). Laxity increases in SB and DB reconstruction were $4.3 \pm 0.9 \mathrm{~mm}$ and $3.2 \pm 0.8$ $\mathrm{mm}$ respectively, and there was also significant difference $(P=0.021)$.

Neither graft pullout from the bone tunnel (fixation failure) nor graft rupture was found during cyclic loading tests. Although the tunnel was created to fit tightly with the graft diameter, gap formations or eccentric position of the graft within the tunnel were found in both SB and DB reconstruction after cyclic loading (Figure 6). Although there was no rupture or maceration of the 
Table 1 Anterior tibial translation and laxity increase during cyclic loading

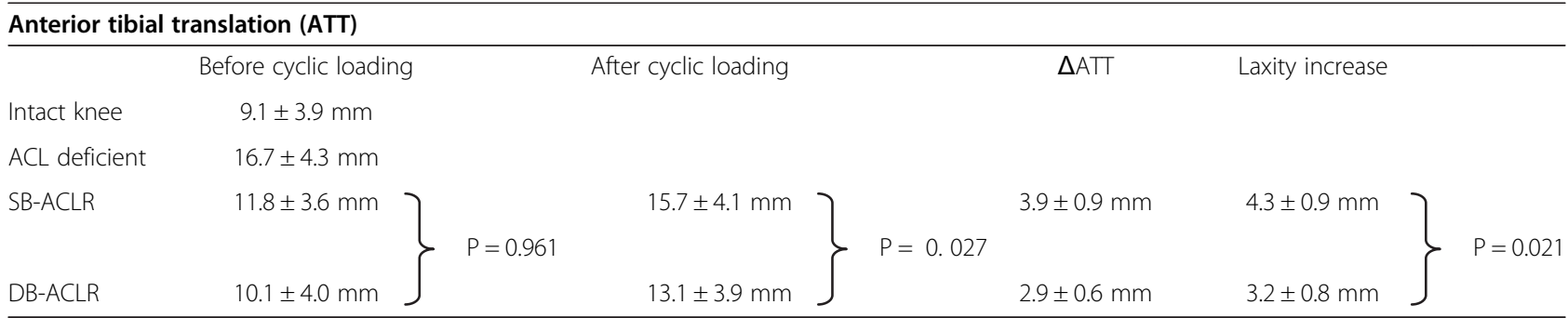

SB-ACLR: single -bundle ACL reconstruction, DB-ACLR: double -bundle ACL reconstruction, $\triangle A T T$ : change in anterior tibial translation.

graft, the graft was deformed at the corner of the femoral tunnel aperture (Figure 7). There was no macroscopic tunnel enlargement found.

\section{Discussions}

The present study suggests that DB reconstruction is superior to SB reconstruction under cyclic load conditions. Repetitive sub-failure loads were applied to the human cadaveric knee and compared early stabilities between SB and DB reconstructed-knees by measuring the laxity increase. While cyclic loading test for ligament reconstruction have been usually performed to compare different types of graft and/or fixation device [24,26], this study compared the same type of graft and fixation device. $\mathrm{DB}$ reconstruction has several biomechanical and biological advantages, including reproduction of functionally different bundle, mimicking native ACL anatomy, increasing graft-tunnel contact area, and reducing stress at the graft-tunnel junction. After 500 cycles of 0 to $100-\mathrm{N}$, the laxity increase for $\mathrm{DB}$ reconstruction was significantly smaller when compared to SB reconstruction, confirming our hypothesis. This is another advantage of DB reconstruction.

The laxity increase consists of the graft slippage from the fixation device and permanent elongation of the

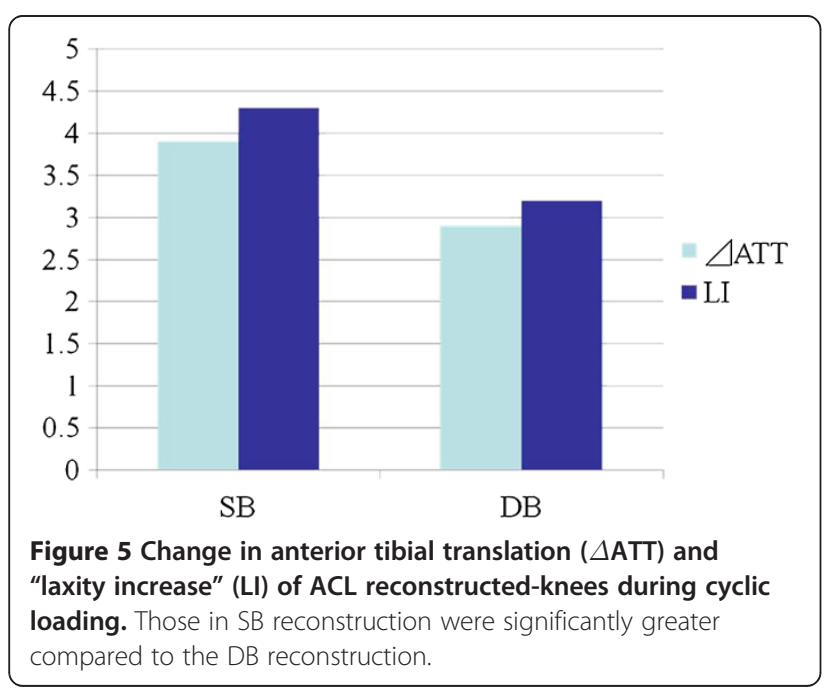

graft construct including plastic deformation of the linkage materials and knot tightening [24,26,27]. Especially in the hamstring tendon, the larger graft elongation appeared to be caused by the longer running route of the hamstring construct due to the placement of the fixation devices, i.e. the Endobutton and the post screw outside of the bone tunnels [28,29]. The main cause of laxity increase is also considered to be due to graft slippage and graft elongation in both SB and DB reconstruction in this study. However, there was significant difference between them. After cyclic loading, the soft tissue graft becomes deformed within the tunnel as it goes the shortest route. A bigger tunnel in SB reconstruction might result in more eccentric positioning of the graft, and with more fixation loss. This may be one of the reasons why SB reconstruction had a larger laxity increase after cyclic loading.

The goal of ACL reconstruction should be to closely restore the native ACL anatomy, which is considered to result in superior clinical outcomes [30]. Therefore, several anatomical studies have been conducted for anatomically accurate ACL reconstruction. Tibial insertion is located at a fossa in front of and lateral to the anterior tibial spine with oval shape [31]. On the other hand, femoral insertion is located at the medial aspect of the lateral femoral condyle just behind the "resident's ridge" [32], in quite a narrow area (Figure 8-a). If this femoral

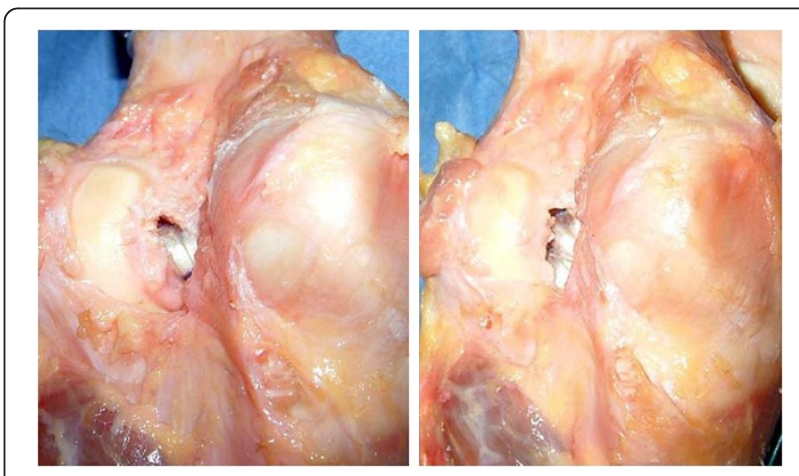

Figure 6 Eccentric graft positioning within femoral tunnels in single-bundle (left) and double-bundle reconstruction (right). Posterior capsule was removed to show graft positioning. 


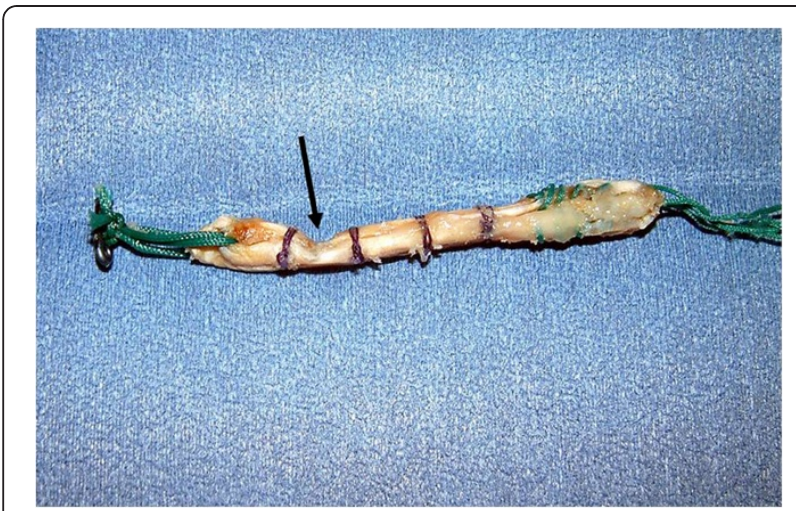

Figure 7 Graft deformation after 500 cycles of 0 to $100-\mathrm{N}$ anterior tibial loads. Graft was deformed at the corner of the femoral tunnel aperture (arrow).

footprint is mimicked, double-tunnels are better than single-tunnel [30]. Of course, a thicker single-graft can cover both the AM and PL bundle footprint. However, such single-graft may deviate into an eccentric position within the large bone tunnel as shown in this study (Figure 8-b). This results in not only stability deterioration but also non-anatomical positioning of the graft. Graft deviations also occur in DB reconstruction, however they are smaller within smaller double tunnels than that in SB reconstruction and grafts are still located within the ACL footprint (Figure 8-c).

Recently the hamstring tendon has become the preferred graft substitute by surgeons who are concerned that harvesting the bone-patellar tendon-bone (BTB) graft may impair the function of the knee extensor mechanism, and hence perform DB reconstruction. Although the hamstring tendon has several advantages making it superior to a BTB graft, it also has several disadvantages. The tendon fixation to the bone is still a challenge in spite of the numerous surgical devices available [33]. Relatively slow maturation of the tendon-bone interface accentuates the need for retaining the graft firmly when the reconstructed-knee is subject to a repetitive load
[34]. In addition, graft deformation within the tunnel is another disadvantage of this graft. On the other hand, the bone plug of the BTB graft may prevent such deviation within the tunnel. This may be one of the reasons why stability after ACL reconstruction with a BTB graft is better than that of the hamstring tendon [35].

There were several limitations in this study. The first was the use of the same knee specimens repeatedly for SB and DB procedures. It is very difficult to obtain fresh cadaver knees in our country, requiring the need to reuse some specimens. Although the reconstructions were performed in random order, mechanical deterioration of the bone might influence results of the second reconstruction. To decrease influence from the first reconstruction, bone plugs packed into the first tunnel were harvested from the femoral condyle of other specimens which had good bone quality. Another limitation was that the average age of the specimens was relatively high. Reduced bone quality of older human subjects predisposes to graft slippage from the fixation device placed within the bone tunnel [36,37]. Although our testing devices permitted 4-DOF, only AP displacements at $30^{\circ}$ of knee flexion were measured. This is a limitation compared to recent advances in technology, such as a robotic manipulator, which can assess 3-dimensional motion at any knee flexion angle. However, we believe that the materials testing machine is more suitable for cyclic loading test because it takes a long time. Thirty degrees of knee flexion was chosen for this biomechanical study, because it is the most critical angle in ACL and tensions of the AM and PL bundle are considered to be equal based on previous biomechanical studies. If another angle was chosen for the cyclic loading test, uneven distribution of forces on the AM and PL bundle would have occurred in DB reconstruction $[4,15]$.

Regardless of these limitations, this study clearly showed a different advantage of $\mathrm{DB}$ reconstruction. If we use a soft-tissue graft, such as a hamstring tendon, the graft deviates within the tunnel after cyclic loading to take the path of shortest distance. This might deteriorate knee
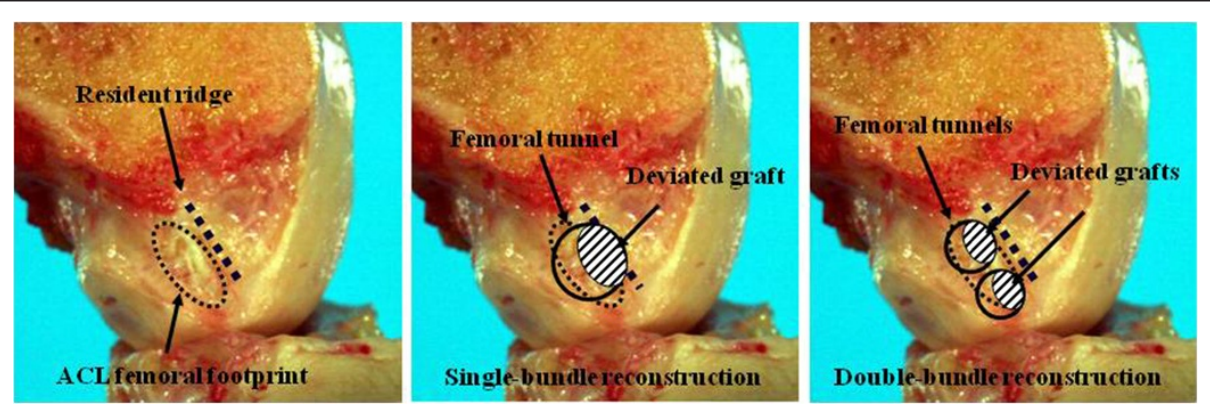

Figure 8 Femoral footprint of $A C L$ and resident ridge. Although a double-bundle graft remains within the femoral footprint, a single-bundle graft might deviate into a non-anatomical position. 
stability after early aggressive rehabilitation [17-21]. Furthermore, a thicker SB graft may end up in a nonanatomical position contrary to the surgeon's intention. We are not stating that $\mathrm{SB}$ is inadequate for $\mathrm{ACL}$ reconstruction, however, surgeons should know the material properties of each graft and choose the optimal graft substitute for each reconstruction technique.

\section{Conclusions}

Although ATT and "laxity increase" was significantly increased in both SB and DB reconstruction with hamstring tendon after cyclic load testing, there was significant difference between them. DB reconstruction might be superior to SB reconstruction in preventing increase in laxity under cyclic loading.

\section{Competing interests}

The authors declare that they have no competing interests.

\section{Authors' contributions}

SN mainly participated in data collection, and drafted the manuscript. Y conceived the main idea, participated in the design of the study, and its revision and coordination. ET participated in the development of the study question and in data collection. YY and HT participated in data collection, and conducted statistical analyses. ST participated in the revision of the manuscript. All authors read and approved the final manuscript.

\section{Received: 12 July 2011 Accepted: 31 May 2012}

Published: 2 July 2012

\section{References}

1. Kocher MS, Steadman JR, Briggs KK, et al: Relationships between objective assessment of ligament stability and subjective assessment of symptoms and function after anterior cruciate ligament reconstruction. Am J Sports Med 2004, 32:629-634

2. Bellier $G$, Christel $P$, Colombet $P$, et al: Double-stranded hamstring graft for anterior cruciate ligament reconstruction. Arthroscopy 2004, 20:890-894.

3. Woo SL, Kanamori A, Zeminski J, et al: The effectiveness of reconstruction of the anterior cruciate ligament with hamstrings and patellar tendon. A cadaveric study comparing anterior tibial and rotational loads. J Bone Joint Surg Am 2002, 84-A:907-914.

4. Yagi M, Wong EK, Kanamori A, et al: Biomechanical analysis of an anatomic anterior cruciate ligament reconstruction. Am J Sports Med 2002, 30:660-666

5. Girgis FG, Marshall JL, Monajem A: The cruciate ligaments of the knee joint. Anatomical, functional and experimental analysis. Clin Orthop Relat Res 1989, 106:216-231.

6. Aglietti P, Giron F, Cuomo P, et al: Single-and double-incision doublebundle ACL reconstruction. Clin Orthop Relat Res 2007, 454:108-113.

7. Järvelä T: Double-bundle versus single-bundle anterior cruciate ligamen reconstruction: a prospective, randomize clinical study. Knee Surg Sports Traumatol Arthrosc 2007, 15:500-507.

8. Muneta T, Koga H, Morito T, et al: A retrospective study of the midterm outcome of two-bundle anterior cruciate ligament reconstruction using quadrupled semitendinosus tendon in comparison with one-bundle reconstruction. Arthroscopy 2006, 22:252-258.

9. Siebold R: Observations on bone tunnel enlargement after doublebundle anterior cruciate ligament reconstruction. Arthroscopy 2007, 23:291-298.

10. Yasuda $\mathrm{K}$, Kondo $\mathrm{E}$, Ichiyama $\mathrm{H}$, et al: Clinical evaluation of anatomic double-bundle anterior cruciate ligament reconstruction procedure using hamstring tendon grafts: comparisons among 3 different procedures. Arthroscopy 2006, 22:240-251.

11. Adachi N, Ochi M, Uchio Y, et al: Reconstruction of the anterior cruciate ligament. Single- versus double-bundle multistranded hamstring tendons. J Bone Joint Surg Br 2004, 86:515-520.
12. Radford WJ, Amis AA: Biomechanics of a double prosthetic ligament in the anterior cruciate deficient knee. J Bone Joint Surg Br 1990, 72:1038-1043.

13. Fujie H, Mabuchi K, Woo SL, et al: The use of robotics technology to study human joint kinematics: a new methodology. J Biomech Eng 1993 115:211-217.

14. Gabriel MT, Wong EK, Woo SL-Y, et al: Distribution of in situ forces in the anterior cruciate ligament in response to rotatory loads. J Orthop Res 2004, 22:85-89.

15. Mae T, Shino K, Miyama T, et al: Single- versus two-femoral socket anterior cruciate ligament reconstruction technique: biomechanical analysis using a robotic simulator. Arthroscopy 2001, 17:708-716.

16. Yamamoto Y, Hsu WH, Woo SL-Y, et al: Knee stability and graft function after anterior cruciate ligament reconstruction. A comparison of a lateral and an anatomic femoral tunnel placement. Am J Sports Med 2004, 32:1825-1832

17. Beynnon BD, Johnson RJ: Anterior cruciate ligament injury rehabilitation in athletes: biomechanical considerations. Sports Med 1996, 22:54-64.

18. Fu FH, Bennett CH, Lattermann C, Ma CB: Current trends in anterior cruciate ligament reconstruction. Part 1: biology and biomechanics of reconstruction. Am J Sports Med 1999, 27:821-830

19. Giurea M, Zorilla P, Amis AA, Aichroth P: Comparative pull-out and cyclicloading strength tests of anchorage of hamstring tendon grafts in anterior cruciate ligament reconstruction. Am J Sports Med 1999, 27:621-625.

20. Höher J, Möller HD, Fu FH: Bone tunnel enlargement after anterior cruciate ligament reconstruction: fact or fiction? Knee Surg Sports Traumatol Arthrosc 1998, 6:231-240.

21. Muneta T, Sekiya I, Ogiuchi T, et al: Effects of aggressive early rehabilitation on the outcome of anterior cruciate ligament reconstruction with multi-strand semitendinosus tendon. Int Orthop 1998, 22:352-6.

22. Woo SL-Y, Orlando CA, Camp JF, et al: Effects of postmortem storage by freezing on ligament tensile behavior. J Biomech 1986, 19:399-404.

23. Race A, Amis AA: Loading of the two bundles of the posterior cruciate ligament: an analysis of bundle function in A-P drawer. J Biomechanics 1996, 29:873-879.

24. Scheffler SU, Südkamp NP, Göckenjan A: Biomechanical comparison of hamstring and patellar tendon graft anterior cruciate ligament reconstruction techniques: The impact of fixation level and fixation method under cyclic loading. Arthroscopy 2002, 18:304-315.

25. Shino K, Mae T, Maeda A, et al: Graft fixation with predetermined tension using a new device, the double spike plate. Arthroscopy 2002, 18:908-911.

26. Höher J, Scheffler SU, Withrow JD, et al: Mechanical behavior of two hamstring graft constructs for reconstruction of the anterior cruciate ligament. J Orthop Res 2000, 18:456-461.

27. Ishibashi Y, Rudy TW, Livesay GA, et al: The effect of anterior cruciate ligament graft fixation site at the tibia on knee stability: evaluation using a robotic testing system. Arthroscopy 1997, 13:177-182.

28. Staerke C, Möhwald A, Gröbel KH, et al: ACL graft migration under cyclic loading. Knee Surg Sports Traumatol Arthrosc 2010, 18:1065-1070.

29. Tsuda E, Fukuda Y, Loh JC, et al: The effect of soft-tissue graft fixation in anterior cruciate ligament reconstruction on graft-tunnel motion under anterior tibial loading. Arthroscopy 2002, 18:960-967

30. van Eck CF, Lesniak BP, Schreiber VM, Fu FH: Anatomic single- and doublebundle anterior cruciate ligament reconstruction flowchart. Arthroscopy 2010, 26:258-268.

31. Arnoczky SP: Anatomy of the anterior cruciate ligament. Clin Orthop Relat Res 1983, 172:19-25.

32. Hutchinson MR, Ash SA: Resident's ridge: assessing the cortical thickness of the lateral wall and roof of the intercondylar notch. Arthroscopy 2003, 19:931-935.

33. Hiraga $Y$, Ishibashi $Y, T$ suda E, et al: Biomechanical comparison of posterior cruciate ligament reconstruction techniques using cyclic loading tests. Knee Surg Sports Traumatol Arthrosc 2006, 14:13-19.

34. Rodeo SA, Kawamura S, Kim HJ, et al: Tendon healing in a bone tunnel differs at the tunnel entrance versus the tunnel exit: an effect of grafttunnel motion? Am J Sports Med 2006, 34:1790-1800.

35. Spindler KP, Kuhn JE, Freedman KB, et al: Anterior cruciate ligament reconstruction autograft choice: bone-tendon-bone versus hamstring. Does it really matter? A systematic review. Am J Sports Med 2004, 32:1986-1995. 
36. Magen HE, Howell SM, Hull ML: Structural properties of six tibial fixation methods for anterior cruciate ligament soft tissue grafts. Am J Sports Med 1999, 27:35-43.

37. Steiner ME, Hecker AT, Brown CHJ, et al: Anterior cruciate ligament graft fixation. Comparison of hamstring and patellar tendon grafts. Am J Sports Med 1994, 22:240-246.

doi:10.1186/1758-2555-4-23

Cite this article as: Nohmi et al:: Biomechanical comparison between single-bundle and double-bundle anterior cruciate ligament

reconstruction with hamstring tendon under cyclic loading condition.

Sports Medicine, Arthroscopy, Rehabilitation, Therapy \& Technology 2012 4:23.

\section{Submit your next manuscript to BioMed Central and take full advantage of:}

- Convenient online submission

- Thorough peer review

- No space constraints or color figure charges

- Immediate publication on acceptance

- Inclusion in PubMed, CAS, Scopus and Google Scholar

- Research which is freely available for redistribution 